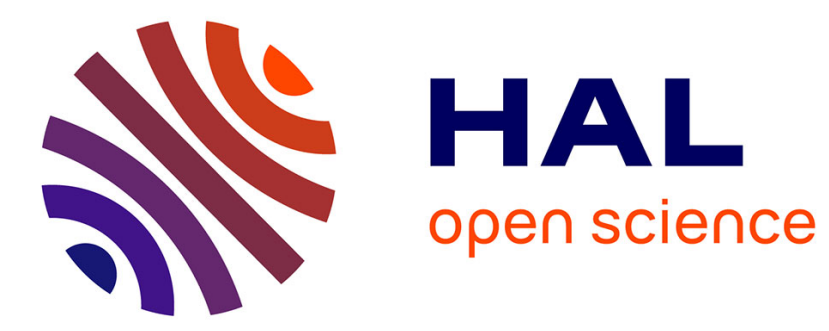

\title{
Ultrasonic Resonance and Relaxation in $\mathrm{RbCl}: \mathrm{OH}$
}

Y. Kogure, W. Ho, A. Granato

\section{To cite this version:}

Y. Kogure, W. Ho, A. Granato. Ultrasonic Resonance and Relaxation in RbCl:OH. Journal de Physique IV Proceedings, 1996, 06 (C8), pp.C8-305-C8-308. 10.1051/jp4:1996866 . jpa-00254674

\section{HAL Id: jpa-00254674 https://hal.science/jpa-00254674}

Submitted on 1 Jan 1996

HAL is a multi-disciplinary open access archive for the deposit and dissemination of scientific research documents, whether they are published or not. The documents may come from teaching and research institutions in France or abroad, or from public or private research centers.
L'archive ouverte pluridisciplinaire HAL, est destinée au dépôt et à la diffusion de documents scientifiques de niveau recherche, publiés ou non, émanant des établissements d'enseignement et de recherche français ou étrangers, des laboratoires publics ou privés. 


\title{
Ultrasonic Resonance and Relaxation in $\mathrm{RbCl}: \mathrm{OH}$
}

\author{
Y. Kogure, W.K.B. Ho* and A.V. Granato* \\ Teikyo University of Science \& Technology, Uenohara, Yamanashi 409-01, Japan \\ * Physics Department, University of Illinois, Urbana, Illinois 61801-3080, U.S.A.
}

\begin{abstract}
The change of elastic constants and mechanical loss from [100] elastic dipoles are calculated on the basis of a six level tunneling model. Resonance and relaxation mechanisms are treated together, and expressions for $C^{\prime}$ - and $C_{11}$ - sound modes are given. The results are used to analyse experimental data for the $\mathrm{RbCl:OH}$ system. The low temperature ultrasonic attenuation is attributed to the resonance mechanism, and the frequency and the concentration dependence are interpreted in terms of dynamical and static interaction between dipoles.
\end{abstract}

\section{INTRODUCTION}

Low temperature mechanical and thermal properties of alkali halide crystals containing hydroxil ions $\left(\mathrm{OH}^{-}\right)$show anomalous behavior due to quantum mechanical tunneling [1]. The resonance absorption of sound wave in glasses has been studied extensively by means of a phenomenological two level system (TLS) model [2]. The $\mathrm{OH}^{-}$ions in alkali halide crystals could be a model system for the TLS. The $\mathrm{OH}^{-}$ions lie on [100] crystal axes and the elastic response could be treated quantitatively by the elastic dipole model. We have developed a full expression for the change of elastic constants due to the elastic dipole. The results are applied to the analysis of our experimental results on $\mathrm{RbCl}: \mathrm{OH}[3]$.

\section{CALCULATION OF ELASTIC CONSTANTS}

A [100] elastic dipole has a tetragonal strain and interacts with external tetragonal stress components. The origin of external stress may be an applied uniaxial stress or internal stress. We choose an $x y z$-coordinate system along the cubic axes. There are 6 allowed states, in which the $\mathrm{OH}^{-}$ion is directed to $x,-x, y,-y, z,-z$ orientations. The Hamiltonian for the dipole under the static external stress can be written as

$$
H^{0}=H^{\mathrm{L}}+H^{\mathrm{T}}+H^{\mathrm{S}}
$$

Where $H^{\mathrm{L}}$ is the lattice hamiltonian and the eigen functions are $\psi_{x}, \psi_{-x}, \psi_{y}, \psi_{-y}, \psi_{z}, \psi_{-z}$. If there are no external stresses (or strains), these six states are equivalent and should have same energies. We may choose the energy to be zero and use sufixes $i$ or $j$ to express these states in general expressions. $H^{\mathrm{T}}$ is the tunneling hamiltonian and $H^{\mathrm{S}}$ corresponds to the interaction between the dipole and the external stress. The interaction energy for the dipole along the $x, y, z$ axis can be expressed as

$$
\begin{aligned}
& S_{x}=-v_{0} \delta \lambda\left(2 \sigma_{x x}-\sigma_{y y}-\sigma_{z z}\right) / 3, \\
& S_{y}=-v_{0} \delta \lambda\left(2 \sigma_{y y}-\sigma_{z z}-\sigma_{x x}\right) / 3, \\
& S_{z}=-v_{0} \delta \lambda\left(2 \sigma_{z z}-\sigma_{x x}-\sigma_{y y}\right) / 3,
\end{aligned}
$$

where $v_{0}$ is the volume occupied by a dipole and $\delta \lambda=\lambda_{1}-\lambda_{2} . \lambda_{1}$ and $\lambda_{2}$ are ellipsoidal strain components produced by a dipole. Then the matrix elements of $H^{0}$ are written as 


$$
H_{i j}^{0}=-\frac{\Delta}{2}\left[\begin{array}{llllll}
0 & 0 & 1 & 1 & 1 & 1 \\
0 & 0 & 1 & 1 & 1 & 1 \\
1 & 1 & 0 & 0 & 1 & 1 \\
1 & 1 & 0 & 0 & 1 & 1 \\
1 & 1 & 1 & 1 & 0 & 0 \\
1 & 1 & 1 & 1 & 0 & 0
\end{array}\right]+\left[\begin{array}{llllll}
S_{x} & 0 & 0 & 0 & 0 & 0 \\
0 & S_{x} & 0 & 0 & 0 & 0 \\
0 & 0 & S_{y} & 0 & 0 & 0 \\
0 & 0 & 0 & S_{y} & 0 & 0 \\
0 & 0 & 0 & 0 & S_{z} & 0 \\
0 & 0 & 0 & 0 & 0 & S_{z}
\end{array}\right]
$$

Here, $\Delta$ is the tunneling energy. Six eigen statess $\Psi_{m}$ and energies $E_{m}$ for the dipole under the external stress are determined from the Schrödinger equation

$$
H^{0} \Psi_{m}=E_{m} \Psi_{m}
$$

by diagonalizing the hamiltonian $H^{0} . \Psi_{m}$ is expanded by $\psi_{i}$ as

$$
\Psi_{m}=\sum_{i} a_{m i} \psi_{i}
$$

There are six eigen states $E_{m}$, three of them $(m=1-3)$ are the solutions of a cubic equation

$$
E^{3}+\left(S_{x} S_{y}+S_{y} S_{z}+S_{z} S_{x}-3 \Delta^{2}\right)+2 \Delta^{3}-S_{x} S_{y} S_{z}=0
$$

and the others are

$$
E_{4}=S_{x}, \quad E_{5}=S_{y}, \quad E_{6}=S_{z} .
$$

As an example, these energy branches under the stress $\left(\sigma_{x x}=0, \sigma_{y y}=\sigma, \sigma_{z z}=-\sigma\right)$ are shown in Figure 1, where the branches shown by dashed lines are inactive to the sound wave.

The matrix elements of the hamiltonian for the interaction between sound wave and elastic dipole, $H_{i j}^{\prime}$ is similar to $H^{\mathrm{s}}$.

$$
H_{i j}^{\prime}=h_{i j} \delta_{i j} \sin (\omega t)
$$

where $\omega$ is the angular frequency of the sound wave. The matrix elements $h_{i j}$ depend on the mode of the sound wave. Two types of the pure sound modes, $C_{11^{-}}$and $2 C^{\prime}\left(=C_{11}-C_{12}\right)$-mode, interact with the $[100]$ dipole. The strain component due to $C_{11}$-mode propagating along the $x$-direction is $\epsilon_{x x}=\epsilon \sin (\omega t)$ and

$$
h_{x x}=-2 q, \quad h_{y y}=q, \quad h_{z z}=q ; \quad q=\left(C_{11}-C_{12}\right) v_{0}(\delta \lambda) \epsilon / 3 .
$$

The strain for the $C^{\prime}$-mode is given by $\epsilon_{x x}=-\epsilon_{y y}=\epsilon / 2$, and the matrix elements are

$$
h_{x x}=-q^{\prime}, \quad h_{y y}=q^{\prime}, \quad h_{z z}=0 ; \quad q^{\prime}=\left(C_{11}-C_{12}\right) v_{0}(\delta \lambda) \epsilon / 2 .
$$

We proceed to calculate the response of the dipole to the sound wave by time dependent perturbation theory. The excess strain produced by dipoles is expressed as

$$
\Delta \epsilon_{i i}=-\frac{2}{3} \frac{n_{0} v_{0} \delta \lambda}{k_{\mathrm{B}} T} \sum_{m} E_{m} \sum_{n(\neq m)} f_{m n} a_{m i} a_{n i}+2 v_{0} \delta \lambda \sum_{m} n_{m}(t) a_{m i}^{2} .
$$

Where,

$$
\begin{gathered}
n_{m}(t)=-\frac{n_{0}}{6 k_{\mathrm{B}} T} b_{m m}\left[\frac{\sin \omega t}{1+\left(\omega \tau_{1}\right)^{2}}+\frac{\cos \omega t}{1+\left(\omega \tau_{1}\right)^{2}}\right] \\
f_{m n}=-\frac{b_{m n}}{2 \hbar}\left[X_{m n}(\omega) \sin \omega t+Y_{m n}(\omega) \cos \omega t\right], \\
X_{m n}(\omega)=\frac{\left(\omega_{m n}-\omega\right) \tau_{2}^{2}}{\left(\omega_{m n}-\omega\right) \tau_{2}^{2}+1}+\frac{\left(\omega_{m n}+\omega\right) \tau_{2}^{2}}{\left(\omega_{m n}+\omega\right)^{2} \tau_{2}^{2}+1}, \\
Y_{m n}(\omega)=\frac{\tau_{2}}{\left(\omega_{m n}-\omega\right) \tau_{2}^{2}+1}+\frac{\tau_{2}}{\left(\omega_{m n}+\omega\right)^{2} \tau_{2}^{2}+1} \\
\omega_{m n}=\left(E_{m}-E_{n}\right) / \hbar, \quad b_{m n}=\sum_{i} a_{m i} a_{n i} h_{i i} .
\end{gathered}
$$




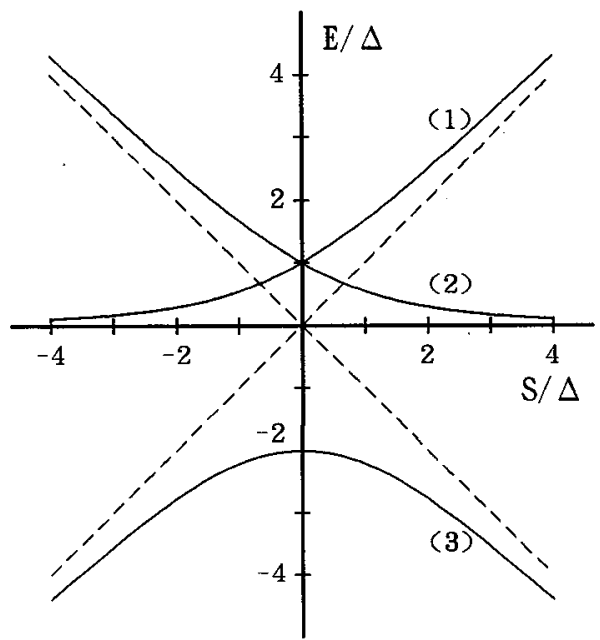

Figure 1: Energy levels under C' stress.
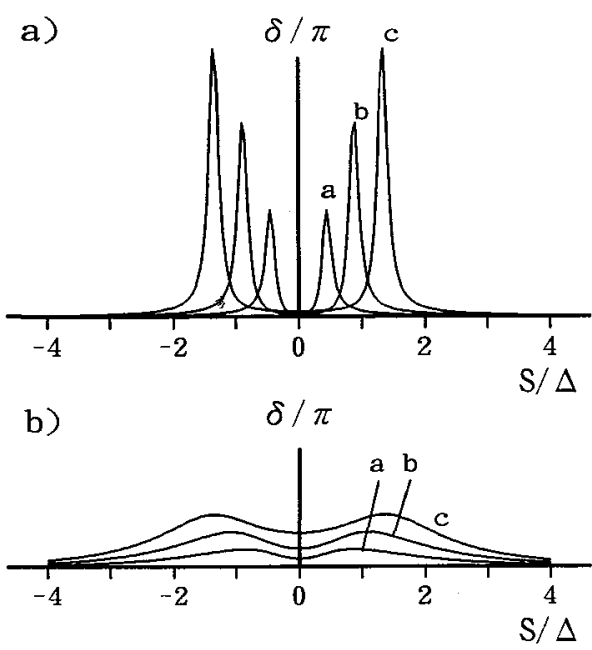

Figure 2: Resonance absorption

Where $\tau_{1}$ is the relaxation time and $\tau_{2}$ is the life time of the dipole. These have similar meanings to the longitudinal and the transverse relaxation time in the theory of magnetic resonance. The first term on the RHS of eq.(11) corresponds to the resonance interaction of the dipoles with the sound wave. The second term corresponds to the relaxation of the dipoles through the change of population $n_{m}$ in the level $m$. The change of elastic constants $\Delta C_{11} / C_{11}$ and $\Delta C^{\prime} / C^{\prime}$ are given by

$$
\Delta C_{11} / C_{11}=\Delta \epsilon_{11} / \epsilon_{11}, \quad \Delta C^{\prime} / C^{\prime}=\left(\Delta \epsilon_{11}-\Delta \epsilon_{12}\right) /\left(\epsilon_{11}-\epsilon_{12}\right) .
$$

As we have assumed the incident sound to be $\epsilon \sin \omega t$, the in-phase component corresponds to the change of sound velocity, and the out-of-phase component corresponds to the absorption or mechanical loss.

As an example, the loss angles $(\delta / \pi)$ for the resonance absorption under the internal stress $S$ are shown in Figure 2, where labels $\mathrm{a}, \mathrm{b}$, and $\mathrm{c}$ represents the sound frequency $\omega=0.5,1.0$ and $1.5 \Delta / \hbar$. When the dipoles have a long life time $\left(\omega \tau_{2}=10\right)$, sharp resonances happen as the energy gap matches to frequency. On the other hand the peak becomes broader when $\tau_{2}$ is small.

\section{RESONANCE AND RELAXATION IN RbCl:OH}

Ultrasonic attenuation and velocity in RbCl:OH system have been measured by the present authors [3]. A typical result is shown in Figure 3. A relaxation peak and the velocity dispersion are seen at around $6 \mathrm{~K}$. Below $3 \mathrm{~K}$ the attenuation increases with decreasing temperatures due to the resonance. The temperature dependence is fitted to the expression

$$
\delta / \pi=A+\Delta_{\mathrm{da}} / T .
$$

The value $\Delta_{\mathrm{da}}$ represents the strength of the resonance absorption. The frequency dependence of $\Delta_{\mathrm{da}}$ for three specimens $(9,15$, and $57 \mathrm{ppm} \mathrm{OH})$ are summarized in Figure 4 . It seen that the frequency dependence is negative $(\propto-0.45)$ for the lower concentration specimens and positive $(\propto .36)$ for the high concentration specimen. This qualitative difference of the resonance absorption may be attributed to the difference of life time $\tau_{2}$. Hess et al. have measured the dielectric absorption in $\mathrm{RbCl}: \mathrm{OH}$ [4], and found that $\tau_{2}$ strongly depends on the $\mathrm{OH}$ concentration due to the interaction between dipoles. $\tau_{2}$ in a $100 \mathrm{ppm}$ specimen was found to be three orders of magnitude smaller than in a $9 \mathrm{ppm}$ specimen.

As shown in Figure 2, resonance absorption shows sharp peaks when $\omega \tau_{2} \gg 1$, then it is sensitive to the distribution of internal stress. The observed negative frequency dependence is possible when the number of dipoles is smaller for higher internal stress. The situation is reasonable if the origins 


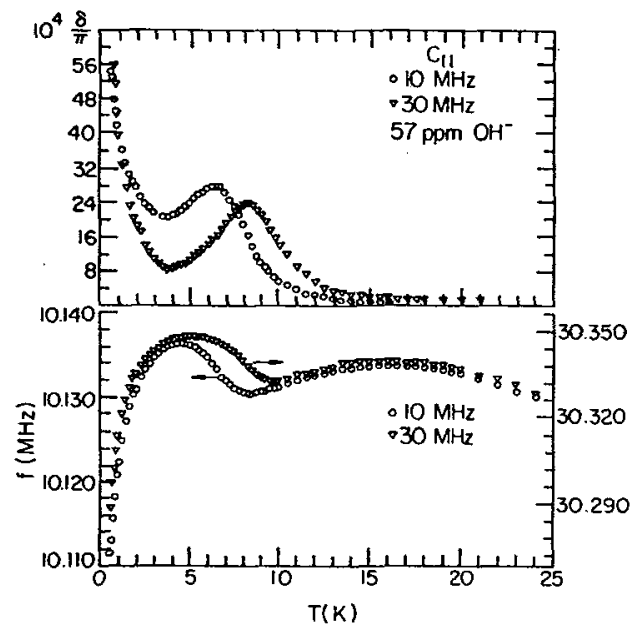

Figure 3: Ultrasonic attenuation and velocity change in $\mathrm{RbCl}: \mathrm{OH}$.

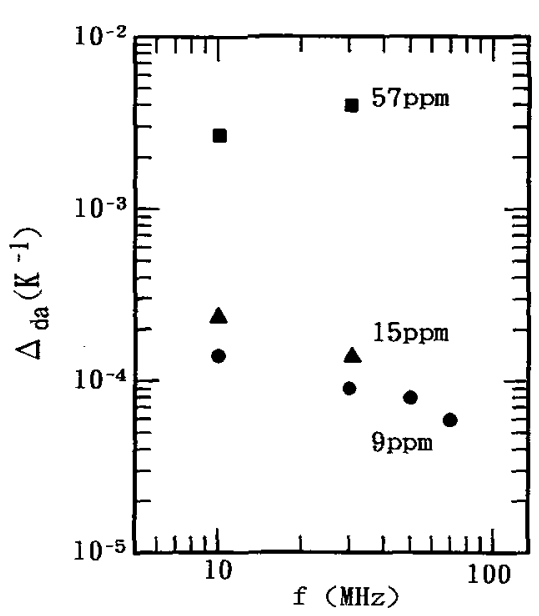

Figure 4: Measured resonance strength $\Delta_{\mathrm{da}}$.

of the internal stress are point defects or dislocations. In the high concentration specimen (57 ppm $\mathrm{OH}) \tau_{2}$ is smaller and the resonance peak becomes broader, then the strength of absorption is less sensitive to the stress distribution and a positive frequency dependence is realized.

The relaxation peak and the velocity dispersion observed at higher temperature are related to the relaxation time $\tau_{1}$. From the analysis of both data, the temperature dependence of $\tau_{1}$ has been determined. The derived $\tau_{1}$ is proportional to $T^{-1}$ and $T^{-4}$ below and above $4 \mathrm{~K}$, respectively. This result suggests that the relaxation is not due to a thermal activation process. $T^{-1}$ and $T^{-4}$ dependences are considered to be due to the one-phonon and the multi-phonon processes.

\section{CONCLUSION}

The resonance and the relaxation phenomena of [100] elastic dipole are treated systematically on the basis of a quantum mechanical tunneling model. RbCl:OH may be a suitable system to test the theory, because it has a proper relaxation time and both phenomena can be observed at different temperatures in a specimen. The frequency and the concentration dependences of the resonance absorption reveal the static interaction between the dipole and the internal stress, and the dynamical dipole-dipole interaction. Similar phenomena are observed in magnetic systems. The energy diagram for the elastic dipole could be determined by scanning the external stress and observing the resonance absorption.

\section{Acknowledgment}

This work was supported in part by the National Science Foundation under grant DMR 93-19773.

\section{References}

[1] Narayanamurti V. and Pohl R.O,, Rev. Mod. Phys. 42 (1970) 201.

[2] Hunklinger S. and Arnold W., in Physical Acoustics, ed. by Mason W.P. (Academic, N.Y., 1976) vol.12, pp.155-215.

[3] Ho W.K.B., Kogure Y. and Granato A.V., Phys. Rev. B 39 (1989) 8154-8161.

[4] Hess H.F. and DeConde K., Phys. Rev. B. 24 (1981) 7419-7422. 